\title{
Calibration System for Multi Channel Oedometer Dong Liu
}

\author{
School of Physics and Telecommunication Engineering, Shaanxi University of Technology, \\ Hanzhong, China \\ 2401452@qq.com
}

Keywords: Oedometer; Calibration system; Automatic; Data acquisition; B/S mode

\begin{abstract}
In order to ensure the accuracy of the experimental data of rock and soil consolidation, the oedometer must be calibrated periodically. A multi channel oedometer calibration system is designed to achieve the control, acquisition, analysis, processing and storage of multiple pressure or lever type oedometer. It can generate automatically test reports, provide remote online calibration data query services with high automation degree of calibration system and simple operation, which can improve the efficiency of the work.
\end{abstract}

\section{Introduction}

Oedometer is soil compression test equipment widely used in engineering construction and engineering survey field. In recent years, the pressure oedometer is gradually replacing the traditional lever type oedometer. The test platform of the air pressure oedometer is composed of a plurality of pressure oedometer. The pressure pump should be controlled and compressed at stated pressure value in the test, so as to obtain the test data by the pressure on soil sample and determine the soil condition by the calculation and analysis. The calibration standard JJF1311-2011[1] required a cyclical mandatory calibration test on oedometer, which can ensure the technical indicators of the consolidation equipment to reach the qualified state and ensure the accuracy of the experimental data.

Therefore, the design of a multi channel oedometer calibration system can calibrate $8 \sim 16$ sets of consolidation at the same time. The output pressure value of each channel pressure oedometer can achieve real-time acquisition, calculation, analysis and judgment consolidation of instrument working qualification, provide calibration data query and generate calibration report automatically, which can improve the work efficiency and record the file's consistency of oedometer test equipment products[2].

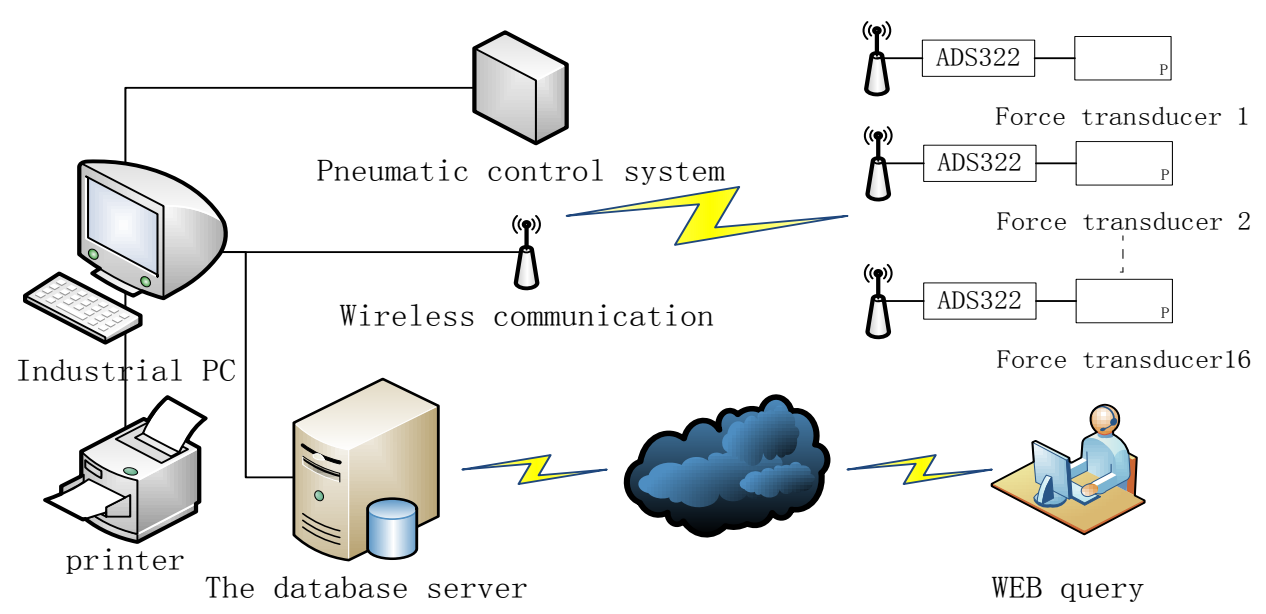

Figure 1. Schematic diagram of calibration system of oedometer

\section{System Structure}

The calibration system of the whole oedometer is composed of industrial control computer, 
DTD46X wireless transmission module, air pressure control system, force sensor, ADS322 data acquisition module, database server and printer. The architecture of the specific design calibration system is shown in Fig. 1.

The control core of the design is the industrial control compute. When pressure oedometer is calibrated, the industrial control machine can control value pressure of air pressure control system on cylinder and affect the force cell in oedometer experimental device. It can also Control wireless transmission module to send the pressure value of the force sensor collected to the PC software of the industrial computer. PC software can display the force sensor data, supply pressure at the controlled calibration, record sensor pressure value, record the complete calibration data, automatically analyze generated test report and upload to the database server. The user can check and calibrate the report online according to the instrument number. When lever type oedometer is calibrated, people should load weights, record data by operating PC program, calibrate points and then load the weights in turn and record the data, calibrate data record, automatically analyze generated test report and upload to the database server. The user can check and calibrate the report online according to the instrument number.

The calibration system of the oedometer calibration system is compatible with the pressure type oedometer and the lever type oedometer, with the characteristics of high degree of automation, safety, reliability, good real-time, remote network query and reports' calibration. It can deal with the shortcoming of the former calibration system, including manual operation, inefficiency and the inconvenient of calibration data management and maintenance.

\section{Hardware Design}

Air Pressure Control System. Air pressure control system is composed of servo motor, reducer, cylinder, piston, screw assembly and digital pressure gauge. PC software control motion controls card to drive servo motor by outputting pulse signal. After the deceleration of reducer, the force is transmitted to the ball screw pair and converted to the piston motion of pushing and pulling by elastic coupling. Piston's position can change the gas pressure in the cylinder. The structure of the pneumatic control system is shown in Fig. 2 [4].

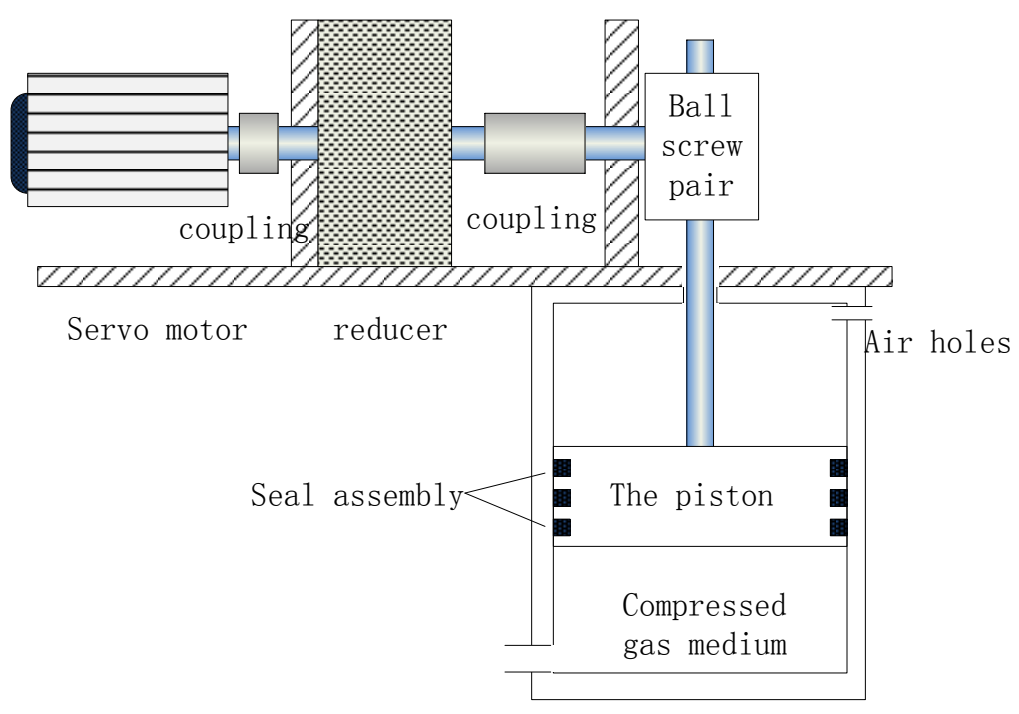

Figure 2. Structure of pressure control

The seal of piston and cylinder is the key of the whole system. Using the combination of GT seal and $\mathrm{O}$ type sealing ring can keep the good sealing and stability in high pressure and high in long time.

The servo motor is used to control the position of the piston of the cylinder and the air pressure is controlled by changing the gas capacity. The incremental PID motor control algorithm is used to 
stabilize the pressure, which can produce accurate and stable pressure. The control precision of the full scale air pressure is $0.02 \%$, which is the first-degree pressure standard precision.

In the literature ${ }^{[4]}$ has introduced that it is a non-linear function in the uniform rotation of the servo motor compression cylinder and cylinder pressure .The pressure is provided no more than 10 pressure points in the calibration of the air pressure oedometer. Therefore, the motor speed and the pressure of the functional relationship are used to control the motor speed of piston rotation and monitor the high precision digital pressure gauge. When the pressure approaches the calibration point, the incremental PID algorithm is used to control the motor stable pressure, stop the motor rotation and calibrate the experiment.

Calibration of Data Acquisition System. Calibration of data acquisition system mainly consists of wireless communication module, data acquisition module and force sensor, as shown in Fig. 1.

The requirement of force cell accuracy is 0.3 in 1311-2011 JJF, so the 0.1 high precision KZY-CZ-3 force sensor is selected, which has smallKZY-CZ-3 temperature drift, good stability, good output linearity, high seal grade of IP65 and can be calibrated in a high humidity environment. The wireless communication module selects the DTX465A module with the RS485 interface protocol. With distance of 200 meters, wireless communication performance is stable and low price to meet the needs of the wireless communication calibration system. The data acquisition module uses module ADS-322 as a force value sensor A/D conversion module, with 24 bit high precision AD conversion chip, RS485 communication interface circuit, data filtering, (diameter $32 \mathrm{~mm}$ ) small volume, light weight (less than $10 \mathrm{~g}$ in single module), which constitutes data acquisition and wireless transmission module with DTX465A modules and selects battery power or a DC power supply. Its comprehensive performance indicators fully meet the calibration system requirements.

The system adopts 17 sets of wireless communication module and unites the communication protocols to 19200 baud rate, parity, 8 data bits and 1 stop bit. The data acquisition wireless transmission module is composed of 16 sets of modules and ADS-322 modules. One set is the industrial control computer receiving module. The industrial control computer adopts the multi channel polling technique, reads instruction and receives its correspondence data by sending address.

Compared to data acquisition card acquisition mode, the wireless distributed acquisition mode can decentralize placement calibration oedometer, reduce the density of centralized data and reduce the interference in the line of the sensor. What's more, the price is more reasonable.

\section{The Software Design}

Calibration Database. In the calibration system of oedometer, it is appreciate to store the information of calibrated apparatus, standard device information in the calibration process, calibration point and pressure value of air pressure oedometer, the calibration point weight of lever type oedometer and calibration real-time data and conclusion analysis in the calibration process. Based on the actual calibration demand, database design is as follows:

Calibration basic information table: \{number, name, specifications, manufacturers, inspection unit\}

Pressure calibration table: \{pressure in the first calibration point... in tenth calibration points \}

Lever calibration table: $\{$ weight in the first lever calibration point ...in tenth calibration points

Calibration data table: \{numbers, the first measurement value in the first point, third measure value in the tenth points\}

Calibration conclusion table: \{number, the first point error... tenth point error, sensitivity, calibration conclusion

The calibration basic information table is used to record the basic information of calibration objects; the pressure calibration table is used to record pressure and the lever calibration table is used to record the calibration point setting value of lever type oedometer. The calibration data table stores the real-time measurement values of the calibration points for three times. Calibration conclusion table stores the analysis of calibration of real-time data, calculation of each calibration point error and conclusion of appropriating. Four tables can be related to the form the corresponding 
detection report conclusions by the calibration equipment.

PC Software Development and Design. Basic Visual 2010 language is used to develop the industrial PC software, which contains four main function modules: parameter setting, oedometer calibration, data maintenance and information four help.

Parameter setting: mainly provides the setting of communication parameters, the motor drive of cylinder control system, the motor control parameter setting, as well as the calibration object and the parameters information setting of the calibration apparatus.

Oedometer calibration: contains fully automatic calibration function of pressure oedometer and manual calibration function of lever type oedometer.

Data maintenance: realizes the calibration information query, maintenance and data calibration and word automatic generation function.

The calibration of the air pressure type oedometer firstly reads the communication parameter information, and sets the wireless communication parameter to detect whether the wireless communication is working properly. If a communication failure occurs, the message will be ejected and the calibration procedure will be exited. If there is no communication failure problem, it will read the parameters sets of calibration point, control the pressure system and provide the corresponding pressure value. If the supply pressure is abnormal, it will be transferred to the error handler. If the supply voltage is stable, the industrial control computer will record the real-time value of each channel force sensor in turn, and then continue the next calibration detection. The detection method is same in each calibration point. After PC program control completes 3 calibration points, the calibration data is transferred into calibration data processing.

On-Line Query System Based on B/S Model. Relatively independent data query system is used to isolate the real-time detection data and on-line query system and to query the system for data maintenance and modification that will not affect the original computer testing data. It is conducive to the safety of data. People can upload the calibration data record of calibration system detection and the analyzed conclusion of the calibration data to the network connection of the database on the server by PC program. They can also visit and query calibration result information though network.

\section{Calibration System Testing and Conclusion}

The multichannel oedometer calibration system is tested and it can achieve high-speed and automated calibration on the air pressure type and the lever type oedometer and provide the function of data' query, processing and report generation. The customer will be able to query the device calibration information through the browser input equipment number.

The application of multi channel oedometer calibration system can effectively improve the calibration efficiency and the accuracy and accuracy of calibration data, which can make up the problems and deficiencies in the calibration of traditional oedometer. There are some shortcomings in the design of calibration system, which will continue to be improved in the process of use.

\section{Acknowledgements}

This thesis is supported by the special research project of Shanxi Provincial Education Department --"the design and development of the calibration information system" (item number: 14JK1135).

\section{References}

[1] JJF 1311-2011 Calibration Specification for Oedometers.China.2011:1-11.

[2] Liu Dong: Design and Development for Calibration Information System of Oedometers (MS. Xi'an Shiyou University,China 2013),p.54.

[3] Guo LI-xiong,NI Jin-quan and JI Hong-lei: Mechanical and Electrical Equipment, Vol.28(2011) No.3,P.22.

[4] HU Yong-jian: Measurement \& Control Technology, Vol.30 (2011) No.8, P. 60. 
[5] Yang Hong-sheng,YaoJin and Yang Zheng-hua.:China Science And Technology Information, Vol.4(2010) ,P.130.

[6] Yu Guo-rui and Lu Fu-min. Shang Hai Measurement And Testing. Vol. 31 (2004) No. 5, P. 50.

[7] Wang Fei,Wang Shuo:China Standardization, Vol.10(2011) ,P.65.

[8] TAO Bairui, GU Ding, MIAO Fengjuan, ZHANG Dongmei, LIU Wenhui and PENG Lizhi: Chinese Journal of Sensors and Actuators, Vol.26 (2013) No.3, P. 435.

[9] ZHANG Yu,PEI Dongxing and ZU Jing: Chinese Journal of Sensors and Actuators. Vol.27 (2011) No .8, P.65.

[10]Li Cong-guan: Technology Innovation a, Vol.3 (2016), P.59. 